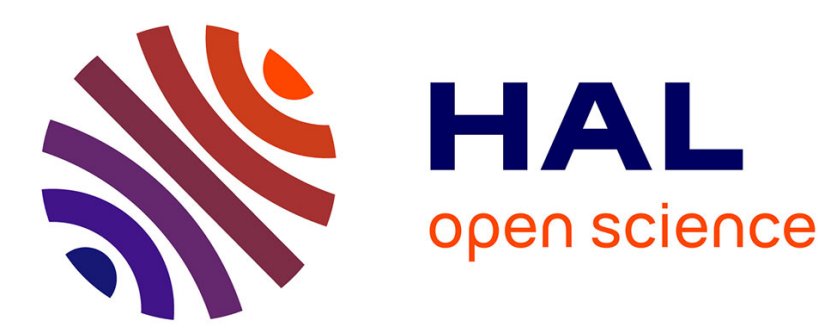

\title{
A combinatorial approach to jumping particles: the parallel TASEP
}

Enrica Duchi, Gilles Schaeffer

\section{To cite this version:}

Enrica Duchi, Gilles Schaeffer. A combinatorial approach to jumping particles: the parallel TASEP. 2005, pp.12. hal-00159636

\section{HAL Id: hal-00159636 \\ https://hal.science/hal-00159636}

Submitted on 5 Jul 2007

HAL is a multi-disciplinary open access archive for the deposit and dissemination of scientific research documents, whether they are published or not. The documents may come from teaching and research institutions in France or abroad, or from public or private research centers.
L'archive ouverte pluridisciplinaire HAL, est destinée au dépôt et à la diffusion de documents scientifiques de niveau recherche, publiés ou non, émanant des établissements d'enseignement et de recherche français ou étrangers, des laboratoires publics ou privés. 


\title{
A COMBINATORIAL APPROACH TO JUMPING PARTICLES: THE PARALLEL TASEP
}

\author{
ENRICA DUCHI* AND GILLES SCHAEFFER ${ }^{\dagger}$
}

\begin{abstract}
In this paper we continue the combinatorial study of models of particles jumping on a row of cells which we initiated with the standard totally asymmetric exclusion process or TASEP (Journal of Combinatorial Theory, Series A, to appear). We consider here the parallel TASEP, in which particles can jump simultaneously. On the one hand, the interest in this process comes from highway traffic modeling: it is the only solvable special case of the Nagel-Schreckenberg automaton, the most popular model in that context. On the other hand, the parallel TASEP is of some theoretical interest because the derivation of its stationary distribution, as appearing in the physics literature, is harder than that of the standard TASEP.

We offer here an elementary derivation that extends the combinatorial approach we developed for the standard TASEP. In particular we show that this stationary distribution can be expressed in terms of refinements of Catalan numbers.
\end{abstract}

RÉSumÉ. L'objet de cet article est de poursuivre l'étude combinatoire d'une famille de modèles de particules sauteuses que nous avons commencé avec le cas du processus d'exclusion totalement asymétrique standard, ou TASEP (Journal of Combinatorial Theory, Series A, to appear). Nous traitons ici le TASEP parallèle, dans lequel les particules peuvent sauter simultanément. L'étude de ce processus est motivée par les nombreux travaux de modélisation du trafic automobile qui portent sur l'automate de Nagel-Schreckenberg: le TASEP parallèle est en effet la seule instance de cet automate stochastique dont la distribution stationnaire soit connue. De plus, le TASEP parallèle présente l'intérêt théorique que la détermination de sa distribution stationnaire par des méthodes de physique mathématique est plus délicate que pour le TASEP standard.

Nous utilisons une approche combinatoire qui étend l'approche que nous avions développée pour le TASEP standard. En particulier nous montrons que cette distribution peut-être décrite en termes de raffinements des nombres de Catalan.

\section{JUMPING PARTICLES AND THE TASEP FAMILY}

The aim of this article is to continue the combinatorial study of a family of models of particles jumping on a row of cells that are known in the physics and probability literature as one dimensional totally asymmetric exclusion processes (TASEPs for short). In order to define TASEPs we first introduce a set of configurations and some rules.

A TASEP configuration is a row of $n$ cells, separated by $n+1$ walls (the leftmost and rightmost ones are borders). Each cell is occupied by one particle, and each particle has a type, black or white (see Figure 1).

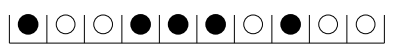

FiguRE 1. A TASEP configuration with $n=10$ cells, 5 black particles, and 5 white particles.

The transitions of the TASEP are based on a mapping $\vartheta$ that modifies a configuration $\tau$ near a wall $i$ to produce a configuration $\vartheta(\tau, i)$. Given a pair $(\tau, i)$ the following rules define its image $\vartheta(\tau, i)$ :

$a$. Rule $\bullet|\circ \rightarrow \circ| \bullet:$ If the wall $i$ separates a black particle (on its left) and a white particle (on its right), then two particles swap to give $\vartheta(\tau, i)$.

$b$. Rule $|\circ \rightarrow| \bullet$ : If the wall is the left border $(i=0)$ and the leftmost cell contains a white particle, this white particle leaves the row and it is replaced by a black particle.

$c$. Rule $\bullet \mid \rightarrow$ o|: If the wall is the right border $(i=n)$ and the rightmost cell contains a black particle, this black particle leaves the row and it is replaced by a white particle.

$d$ In the other cases, nothing happens, $\vartheta(\tau, i)=\tau$.

Date: November 22, 2004.

* Supported by a post-doctoral grant of the CNRS.

† Supported in part by EC's IHRP Programm, within the Research Training Network Algebraic Combinatorics in Europe, grant HPRN-CT-2001-00272. 


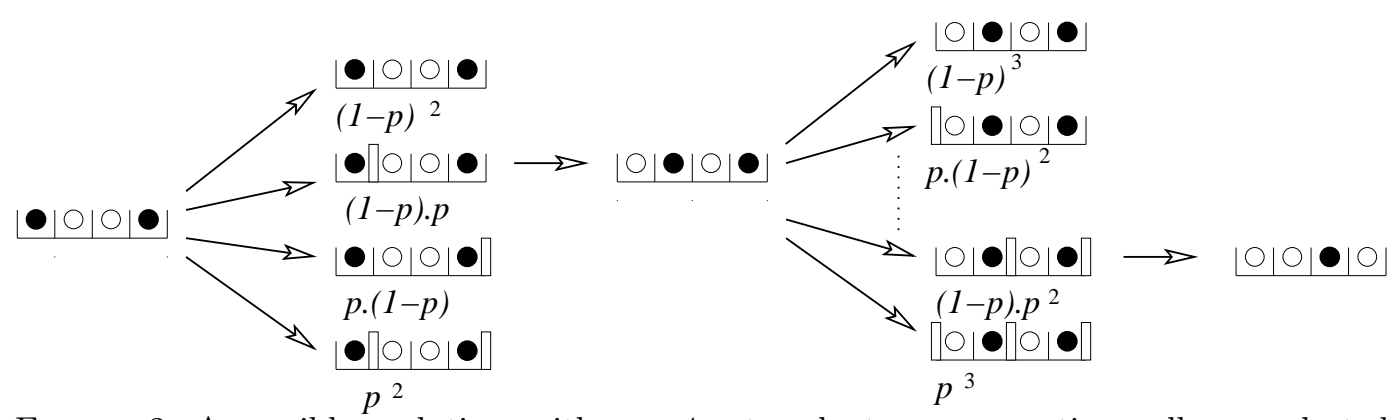

Figure 2. A possible evolution, with $n=4$ : at each step, some active walls are selected with the given probabilities, and they trigger a transition.

Given a configuration $\tau$, let $M(\tau)$ be the set of walls on which the previous application $\vartheta$ effectively does something: inner walls with a neighborhood of the form $\bullet \mid \circ$, or borders with a neighborhood of the form $\mid \circ$ or $\bullet$. The definition of $\vartheta$ can be extended to any subset $\left\{i_{1}, \ldots, i_{k}\right\}$ of $M(\tau)$ by setting $\vartheta\left(\tau, i_{1}, \ldots, i_{k}\right)=$ $\vartheta\left(\vartheta\left(\tau, i_{1}, \ldots, i_{k-1}\right), i_{k}\right)$. Observe that this extended application can be interpreted as performing moves at walls $i_{1}, \ldots, i_{k}$ in parallel since the basic application acts only locally and $M(\tau)$ never contains two adjacent walls. A pair $(\tau, A)$ with $A \subset M(\tau)$ will be referred to as an active configuration, and from now on $\vartheta$ is considered as a mapping from the set of active configurations into the set of TASEP configurations.

In the previous work [3], we dealt with several variants of sequential TASEPs. In particular, the standard sequential TASEP with open boundaries is a Markov chain $S^{\text {seq }}$ on the set of TASEP configurations with $n$ cells whose dynamic is defined as follows in terms of $\vartheta$ :

- Let $\tau=S^{\operatorname{seq}}(t)$ be the current configuration.

- Choose a uniform random wall $i$ in $\{0, \ldots, n\}$.

- Set $S^{\operatorname{seq}}(t+1)=\vartheta(\tau, i)$.

The aim of the present article is to extend our approach to a more general model in which particles are allowed to jump simultaneously: the parallel TASEP is a Markov chain $S / /$ on the set of TASEP configurations whose dynamic is defined as follows in terms of $\vartheta$ :

- Let $\tau=S / /(t)$ be the current configuration, $M=M(\tau)$, and $m=m(\tau)=|M|$.

- Choose a random subset $A$ of $M$ by independently giving to each wall of $M$ probability $p$ to be taken. In other terms, the probability that $A=\left\{i_{1}, \ldots, i_{k}\right\}$ for some given distinct elements $i_{1}, \ldots, i_{k}$ of $M$ is $p^{k}(1-p)^{m-k}$. The walls in $A$ are referred to as the active walls of the active configuration $(\tau, A)$. - Then set $S^{/ /}(t+1)=\vartheta(\tau, A)$.

Figure 2 illustrates the application of these rules, with active walls appearing as $\|$. Observe that the transformation $\vartheta$ makes black particles travel from left to right, and makes white particles do the opposite.

The difference between the sequential and the parallel TASEP is thus that in the first process, only one wall can trigger a move at a time, while in the second simultaneous moves can occur. Observe that if $p$ is very small, it is unlikely that more than one particle jump at a time (since $p^{2} \ll p$ ). This implies that in the limit $p \rightarrow 0$, the parallel TASEP reduces to a (very slow) sequential TASEP.

We got interested in the TASEP because Derrida et al. $[1,2]$ proved that the stationary distribution of this Markov chain involves Catalan numbers. In [3], we gave a combinatorial explanation of this fact. In the present paper we extend our combinatorial approach to derive the stationary distribution of the parallel TASEP. Although we concentrate here on TASEPs with open boundary conditions, it is worth indicating that variants with periodic boundaries can be defined and studied similarly (identifying walls 0 and $n$ and concentrating on rule $a$ ).

The stationary distribution of the parallel TASEP was first obtained by Schadschneider et al. (see [6] and ref. therein) in the easier case of periodic boundaries and by Evans et al. [4] in the case with open boundaries. This last derivation is based on the same matrix ansatz approach developed by Derrida et al. for the sequential TASEP [2], but requires the introduction of a quartic (instead of quadratic) algebra. This extra complexity reflects in our combinatorial approach, in the sense that, with respect to [3], new 
ingredients are necessary to construct the covering Markov chain on which we rely. However a nice feature of our approach is that we are able to remain within the realm of Catalan combinatorial structures.

To conclude this introduction, it is worth stressing the fact that the determination of the stationary distribution allows to compute some physical quantities related to the model (densities, flows, phase diagrams,...). Our approach, while providing a new derivation and interpretation of the stationary distribution, leads then to the same computations as far as these next steps are concerned. We thus do not reproduce the corresponding discussions.

\section{The COMBinAtorial APPROACH}

Our method to study the TASEP consists in the construction of a new covering Markov chain $X^{/ /}$on a set $\Omega_{n}$ of complete configurations, that satisfies two main requirements: on the one hand the stationary distribution of the TASEP chain $S / /$ can be simply expressed in terms of that of the covering chain $X^{/ /}$; on the other hand the stationary distribution of the covering chain $X / /$ can be expressed by means of a combinatorial parameter defined on the set $\Omega_{n}$.

2.1. The complete Markov chain. Since the parallel TASEP $S$ // yields back the sequential TASEP $S^{\text {seq }}$ for $p \rightarrow 0$, we first try to adapt directly the construction of [3] to simultaneous jumps.

Following [3], define a complete configuration of $\Omega_{n}$ to be a pair of rows of particles satisfying the following constraints: $(i)$ there is an equal number of black and white particles (the balance condition); (ii) on the left hand side of any vertical wall there are at least as many black particles as black ones (the positivity condition). An example of complete configuration is given in Figure 3. The number of elements of $\Omega_{n}$ is the $n$th Catalan number $\frac{1}{n+1}\left(\begin{array}{c}2 n \\ n\end{array}\right)$ (see [3], although readers with a background in combinatorics may as well recognize directly bicolored Motzkin paths in disguise).

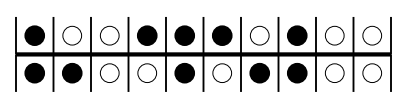

Figure 3. A complete configuration with $n=14$.

Given a configuration $\omega$ of $\Omega_{n}$, let top $(\omega)$ denote its first row, which is a TASEP configuration. Still in the steps of [3], we look for a covering chain $X / /$ on $\Omega_{n}$ that mimics in the top row the TASEP $S / /$. More precisely we would like to define $X^{/ /}$exactly as $S / /$ with $\vartheta$ replaced by a mapping $T$ that has nice combinatorial properties and that extends $\vartheta$ in the following sense: given a configuration $\omega$ of $\Omega_{n}$ and a set of active walls $A, T$ should produce a new configuration $\omega^{\prime}$ of $\Omega_{n}$ in such a way that if $\operatorname{top}(\omega)=\tau$, then $\operatorname{top}\left(\omega^{\prime}\right)=\vartheta(\tau, A)$.

However such a direct extension of the construction of [3] does not seem to be sufficient to account for the more complex dynamic of the parallel TASEP. Instead we need to introduce a further randomization step that is conveniently described in terms of colors.

A well colored configuration $(\omega, A, R, G)$ consists of a complete configuration $\omega$, a set $A$ of active walls, a set $R$ of red walls and a set $G$ of green walls, such that:

- the set $A$ of active wall is a subset of $M(\omega)=M(\operatorname{top}(\omega))$, the set of walls around which the local configuration in the first row is $\bullet|\circ,| \circ$ or $\bullet \mid$,

- the set $R$ of red walls and the set $G$ of green walls form a partition $(R, G)$ of the subset $C(\omega, A)$ of $A$ consisting of walls around which the local configuration is $: 1_{0}^{\circ}$.

We now define a parameter $Q$ on complete configurations and its "randomized" version $q$ on the set of well colored configurations. Given $(\omega, A, R, G)$ a well colored configuration, we set

$$
Q(\omega)=(1-p)^{h(\omega)-m(\omega)}, \quad \text { and } \quad q(\omega, A, R, G)=p^{|A|}(1-p)^{h(\omega)-|A|} \cdot p^{|R|}(1-p)^{|G|},
$$

where $h(\omega)$ denote the number of columns of the form $\left.\right|_{\bullet} ^{\bullet}|,|_{0}^{\bullet} \mid$ and $\left.\right|_{\bullet} ^{0} \mid$ in $\omega$, and $m(\omega)=|M(\omega)|$. A configuration $\omega$ contains the same number of black and white particles, so that $h(\omega)$ can be rewritten in 
various ways:

$$
\begin{aligned}
h(\omega) & =\mid\left\{\text { columns }\left.\right|_{\circ} ^{\bullet} \mid \text { or }\left|\begin{array}{c}
\circ \\
\bullet
\end{array}\right|\right\}\left|+\frac{1}{2} \cdot\right|\left\{\text { columns }|\bullet| \text { or }\left.\right|_{\circ} ^{\circ} \mid\right\} \mid \\
& =\frac{n}{2}+\frac{1}{2} \cdot \mid\left\{\text { columns }\left.\right|_{\circ} ^{\bullet} \mid \text { or }\left|\begin{array}{c}
\bullet \\
\bullet
\end{array}\right|\right\}|=n-|\left\{\text { columns }\left|\begin{array}{l}
0 \\
\circ
\end{array}\right|\right\} \mid .
\end{aligned}
$$

Again, readers with a background in enumerative combinatorics will recognize the statistic $h(\omega)$ as $n / 2$ plus half of the number of horizontal steps in the bicolored Motzkin path associated to the configuration $\omega$.

Observe that

$$
q(\omega, A, R, G)=Q(\omega) \cdot p^{|A|}(1-p)^{m(\omega)-|A|} \cdot p^{|R|}(1-p)^{|G|} .
$$

Given $\omega$, we can thus apply the binomial formula $\sum_{U \subset V} x^{|U|} y^{|V|-|U|}=(x+y)^{|V|}$ to sum over all partitions $(R, G)$ of $C(\omega, A)$, and then again to sum over all subsets $A \subset M(\omega)$ :

$$
\sum_{A, R, G} q(\omega, A, R, G)=Q(\omega),
$$

where the summation is over all triples $(A, R, G)$ such that $(\omega, A, R, G)$ is a well colored configuration.

The key of our combinatorial approach is that we can construct an application $T$ that behaves nicely with respect to the parameter $q$. The construction is given in Section 3.

Theorem 1. There is a bijection $\bar{T}$ from the set of well colored configurations onto itself such that:

- The mapping $T$, defined as the first component of $\bar{T}$, mimics in the top row the mapping $\vartheta$. More explicitly, if $\left(\omega^{\prime}, A^{\prime}, R^{\prime}, G^{\prime}\right)=\bar{T}(\omega, A, R, G)$ is the image of a well colored configuration $(\omega, A, R, G)$ by $\bar{T}$, then $\omega^{\prime}=T(\omega, A, R, G)$ satisfies

$$
\operatorname{top}\left(\omega^{\prime}\right)=\vartheta(\operatorname{top}(\omega), A) .
$$

- The parameter $q$ is preserved by the bijection $\bar{T}$ : for any well colored configuration $(\omega, A, R, G)$,

$$
q(\bar{T}(\omega, A, R, G))=q(\omega, A, R, G) .
$$

Observe that the first property of $\bar{T}$ completely defines its action on the first row of configurations: in particular, $T$ must move black particles from left to right in the first row. We shall see in Section 3 , when we explicitly describe $\bar{T}$ that in the second row it will move black particles in the opposite direction, from right to left.

With Theorem 1 at hand, we are in the position to define our Markov chain $X^{/ /}$on the set $\Omega_{n}$ :

- Let $\omega=X^{/ /}(t)$ be the current configuration, $M=M(\omega)=M(\operatorname{top}(\omega))$, and $m=m(\omega)=|M|$.

- Choose a random subset $A$ of $M$ by independently giving to each wall of $M$ probability $p$ to be taken. In other terms, the probability that $A=\left\{i_{1}, \ldots, i_{k}\right\}$ for some given distinct elements $i_{1}, \ldots, i_{k}$ of $M$ is $p^{k}(1-p)^{m-k}$. The walls in the set $A$ are referred to as active walls, and the pair $(\omega, A)$ as an active configuration.

- Next, let $C=C(\omega, A)$ be the subset of $A$ consisting of walls around which the local configuration is - $\left.\right|_{\circ} ^{\circ}$. Then each wall of $C$ is colored red with probability $p$ or green with probability $1-p$. In other terms we randomly partition $C$ into $R$ (red walls) and $G$ (green walls), and associate to the active configuration $(\omega, A)$ a well colored configuration $(\omega, A, R, G)$.

- Then set $X^{/ /}(t+1)=T(\omega, A, R, G)$.

See Figure 4 for an illustration.

Let us compare the dynamic of $X^{/ /}$and $S /$. In the chain $X^{/ /}$, a supplementary random coloring step is performed that does not exist in the chain $S / /$. In particular we allow the action of $T$ to depend on this distinction between colors, and this will be used in the actual construction of the mapping $T$ in Section 3 . However the colors only affect the bottom row: the action of $T$ on the top row depends only on $A$ and, as already indicated, mimics $\vartheta$. As a consequence, if one only considers the top row, the coloring step in the definition of $X^{/ /}$can be ignored, and we obtain the following relation between $X^{/ /}$and $S / /$.

Proposition 1. The chains $S / /$ and top $\left(X^{/ /}\right)$have the same dynamics and the same stationary distributions. 


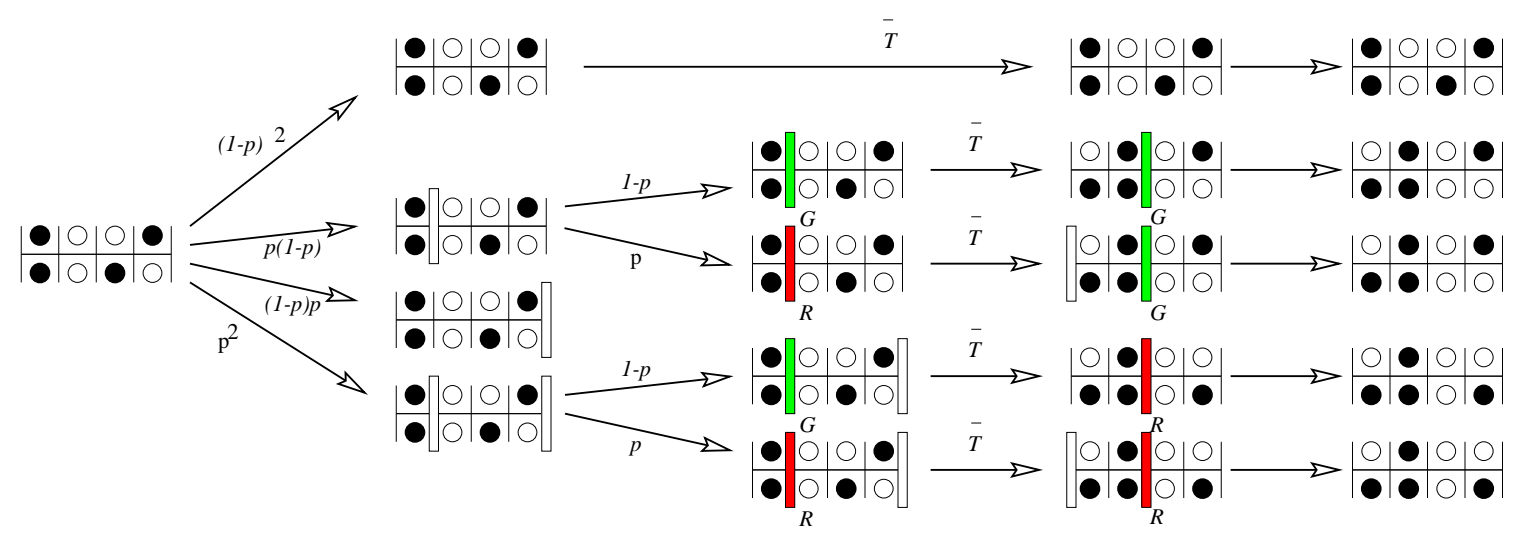

FiguRE 4 . The possible transitions for a configuration $\omega$ with $M(\omega)=\{1,4\}$.

2.2. The stationary distribution. As we shall see in Section 3, the transitions of the complete chain $X / /$ that originate from exactly one wall and such that this wall is not red are exactly the transitions of the chain $X$ studied in [3] in relation with the sequential TASEP. The chain $X$ was proved irreducible there and this implies that the chain $X^{/ /}$, that has more transitions, is irreducible as well. Moreover there is a positive probability to stay in any configuration, so that the chain $X / /$ is aperiodic. A classical result of the theory of finite Markov chain is that an irreducible aperiodic chain has a unique stationary distribution to which it converges [5]. Our main result is then the following theorem.

Theorem 2. The stationary distribution of the Markov chain $X / /$ is proportional to the parameter

$$
Q(\omega)=(1-p)^{h(\omega)-m(\omega)},
$$

where $h(\omega)$ is the number of columns of the form $\left.\left.\right|_{\bullet} ^{\bullet}||\right|_{0} ^{,\bullet} \mid$ or $\left.\right|_{\bullet} ^{0} \mid$ and $m(\omega)$ the number of walls at which a transition could occur in the first row (i.e walls around which the local configuration in the top row is $\bullet|\circ, \bullet|$ or $(\circ)$. In other terms,

$$
\operatorname{Prob}\left(X^{/ /}(t)=\omega\right) \underset{t \rightarrow \infty}{\longrightarrow} \frac{1}{Z_{n}(1-p)}(1-p)^{h(\omega)-m(\omega)} \quad \text { where } \quad Z_{n}(x)=\sum_{\omega \in \Omega_{n}} x^{h(\omega)-m(\omega)} .
$$

In particular $Z_{n}(x)$ is a combinatorial refinement of the Catalan numbers $Z_{n}(1)=\frac{1}{n+1}\left(\begin{array}{c}2 n \\ n\end{array}\right)$.

An immediate consequence of this theorem is the following interpretation of the stationary distribution of the Markov chain $S / /$ in terms of weighted complete configurations with fixed top row.

Theorem 3. The stationary distribution of the Markov chain $S / /$ is proportional to

$$
\pi(\tau)=\sum_{t o p(\omega)=\tau}(1-p)^{h(\omega)-m(\tau)} .
$$

Let a balanced substring of $\tau$ be a subword $\sigma=\tau_{i_{1}} \tau_{i_{2}} \ldots \tau_{i_{2 r}}$ of $\tau$ that is a balanced parenthesis word with black and white particles respectively viewed as opening and closing parentheses. In this case write $\sigma \vdash \tau$ and $|\sigma|=r$. Then $\pi(\omega)$ can be rewritten as

$$
\pi(\tau)=(1-p)^{n-m(\tau)} \sum_{\sigma \vdash \tau}(1-p)^{-|\sigma|} .
$$

For instance, for a configuration of the type $\circ \cdots \circ \bullet \cdots \bullet$, we obtain

$$
\pi(\underbrace{0 \cdots \bullet}_{k} \underbrace{\bullet \cdots \bullet}_{n-k})=(1-p)^{n-3}, \quad \text { for all } 0<k<n,
$$

which is independent of $k$, whereas for $\bullet \cdots \bullet \cdots \cdots$, the probability depends on $k$ as follows:

$$
\pi(\underbrace{\bullet \cdots \bullet}_{k} \underbrace{\circ \cdots \circ}_{n-k})=\sum_{r=0}^{\min (k, n-k)}\left(\begin{array}{c}
k \\
r
\end{array}\right)\left(\begin{array}{c}
n-k \\
r
\end{array}\right)(1-p)^{n-1-r}, \quad \text { for all } 0<k<n .
$$


Corollary 1. In the limit $p=0$, we recover the stationary distribution of the sequential TASEP: indeed, for $p=0$, we get $Q(\omega)=1$ for all $\omega$, and $Z_{n}(1)=\left|\Omega_{n}\right|=\frac{1}{n+1}\left(\begin{array}{c}2 n \\ n\end{array}\right)$ :

$$
\operatorname{Pr}\left(S^{s e q}=\tau\right)=\frac{\mid\{\omega \mid \text { top }(\omega)=\tau\} \mid}{\frac{1}{n+1}\left(\begin{array}{c}
2 n \\
n
\end{array}\right)} .
$$

2.3. Proof of the stationarity. Our aim is to show that the unnormalized distribution $Q(\omega)$ is stationary. Let us thus assume that $\operatorname{Prob}\left(X^{/ /}(t)=\omega\right)=c_{0} Q(\omega)$ for some constant $c_{0}$ and all $\omega \in \Omega_{n}$, and let us $\operatorname{compute} \operatorname{Prob}\left(X^{/ /}(t+1)=\omega^{\prime}\right)$.

By construction, the probability to be in configuration $\omega^{\prime}$ at time $t+1$ is the sum over the probability to be at time $t$ in a configuration $\omega$ multiplied by the probability to select subsets $A, R$, and $G$ such that $T(\omega, A, R, G)=\omega^{\prime}$. More precisely, the probability to select $A$ from $M(\omega)$ is $p^{|A|}(1-p)^{m(k)-|A|}$, and the probability to select $R$ and $G$ is $p^{|R|}(1-p)^{|G|}$. Hence

$$
\begin{aligned}
\operatorname{Prob}\left(X^{/ /}(t+1)=\omega^{\prime}\right) & =\sum_{(w, A, R, G) \in T^{-1}\left(\omega^{\prime}\right)} \operatorname{Prob}\left(X^{/ /}(t)=\omega\right) p^{|A|}(1-p)^{m(\omega)-|A|} p^{|R|}(1-p)^{|G|}, \\
& =\sum_{(w, A, R, G) \in T^{-1}\left(\omega^{\prime}\right)} c_{0} q(w, A, R, G),
\end{aligned}
$$

where the second line follows from the hypothesis $\operatorname{Prob}\left(X^{/ /}(t)=\omega\right)=c_{0} Q(\omega)$ and from Formula (1).

In view of Theorem $1, T$ is the first component of the bijection $\bar{T}$, so that $T^{-1}\left(\omega^{\prime}\right)$ is the inverse image by $\bar{T}$ of the set of well colored configurations of the form $\left(\omega^{\prime}, A^{\prime}, R^{\prime}, G^{\prime}\right)$. This implies

$$
\operatorname{Prob}\left(X^{\prime \prime}(t+1)=\omega^{\prime}\right)=\sum_{A^{\prime}, R^{\prime}, G^{\prime}} c_{0} q\left(\bar{T}^{-1}\left(w^{\prime}, A^{\prime}, R^{\prime}, G^{\prime}\right)\right)=\sum_{A^{\prime}, R^{\prime}, G^{\prime}} c_{0} q\left(w^{\prime}, A^{\prime}, R^{\prime}, G^{\prime}\right)=c_{0} Q\left(\omega^{\prime}\right) .
$$

where the summations are over all triples $\left(A^{\prime}, R^{\prime}, G^{\prime}\right)$ such that $\left(\omega^{\prime}, A^{\prime}, R^{\prime}, G^{\prime}\right)$ is a well colored configuration, the second equality follows from the invariance of $q$ under the action of $\bar{T}$, as stated in Theorem 1 , and the last equality is Formula (2).

\section{THE BIJECTION $\bar{T}$}

In this section we prove Theorem 1 by describing a bijection $\bar{T}$ that transports the parameter $q$. We first give the definitions of some local operations, and use them to describe an intermediate mapping $\psi$. Finally we present $\bar{T}$ and check that $\bar{T}$ satisfied the requirements.

3.1. Local operations. We shall use two types of local operations: deletions map configurations of $\Omega_{n}$ to configurations of $\Omega_{n-1}$, while insertions map configurations of $\Omega_{n-1}$ to configurations of $\Omega_{n}$. In the following definitions, the numbering of walls always refers to the configuration of $\Omega_{n}$ :

- A right deletion at $i \neq 0$ consists in

- if $i \neq n$, removing a $\left.\right|_{\bullet} ^{\circ} \mid$-column on the right of $i$, that is: $\left.\omega_{1}\right|_{x} ^{y} \|_{\bullet}^{\circ}\left|\omega_{2} \mapsto \omega_{1}\right|_{x}^{y} \mid \omega_{2}$.

- if $i=n$, removing a $\left.\right|_{0} ^{\bullet} \mid$-column at the right border, that is: $\left.\omega_{1}\right|_{\circ} ^{\bullet} \|_{n} \mapsto \omega_{1} \mid$.

- A left deletion at $i \neq n$ consists in

- if $i \neq 0$, removing a $\left.\bullet\right|_{\circ}$-diagonal around $i$, that is: $\left.\omega_{1}\right|_{x} ^{\bullet} \|_{i^{\circ}}^{y}\left|\omega_{2} \mapsto \omega_{1}\right|_{x}^{y} \mid \omega_{2}$.

- if $i=0$, removing a $\left.\right|_{\bullet} ^{\circ} \mid$-column on the left border, that is: $\|_{0}^{\circ}\left|\omega_{2} \mapsto\right| \omega_{2}$.

- A right insertion at $j \neq n$ consists in

- if $j \neq 0$, inserting a $\left.\right|_{\bullet} ^{0} \mid$-column on the right of wall $j$, that is: $\left.\omega_{1}\right|_{x} ^{y}\left|\omega_{2} \mapsto \omega_{1}\right|_{x}^{y} \|_{j}^{0} \mid \omega_{2}$.

- if $j=0$, inserting a $\left.\right|_{\bullet} ^{0} \mid$-column on the left border, that is: $\left|{ }_{x}^{y}\right| \omega_{2} \mapsto \|_{0}^{0} \bullet{ }_{x}^{y} \mid \omega_{2}$.

- A left insertion at $j \neq 0$ consists in

- if $j \neq n$, inserting a $\left.\bullet\right|_{\circ}$-diagonal around column $j$, that is: $\left.\omega_{1}\right|_{x} ^{y}\left|\omega_{2} \mapsto \omega_{1}\right|_{x}^{\bullet} \|_{j}^{y} \mid \omega_{2}$.

- if $j=n$, inserting a $\left.\right|_{0} ^{\bullet} \mid$-column at the right border, that is: $\left.\left.\omega_{1}\right|_{x} ^{y}\left|\mapsto \omega_{1}\right|_{x}^{y}\right|_{0} ^{\bullet} \|_{n}^{j}$.

In the case of the sequential TASEP [3], we used these operations to construct a bijection from the set of complete configuration with exactly one active wall, onto itself. The bijection essentially consisted in applying one deletion at the active wall and one insertion at another nearby wall. Since the main difference 
between the sequential and the parallel TASEP is the fact that there may be several active walls, one could just try to apply the bijection of [3] to all these walls in parallel. However, this naive approach fails when there are two active walls that are too close, because the transformations applied to nearby walls may interfere.

In order to circumvent this ambiguity a new operation is needed:

- A block deletion at a wall $i$ of type $\left.\right|_{\bullet} ^{\bullet} \|_{i^{\circ}}^{\circ} \mid$ consists in removing the block around $i: \omega_{1}\left|\stackrel{\bullet}{\bullet} \|_{i^{\circ}}^{\circ}\right| \omega_{2} \mapsto \omega_{1} \mid \omega_{2}$.

Using the corresponding insertion, it would be possible to describe the bijection $\bar{T}$ directly as the simultaneous application of some deletions and insertions near active walls. It will however prove more convenient to give a sequential description in terms of a partial application $\psi$.

3.2. The mapping $\psi$. Given a configuration $(\omega, A, R, G)$, a pointer is an element of $A \cup\{\perp, \top\}$. The value $\perp$ and $T$ are respectively interpreted as positions of the pointer to the left and to the right of the configuration. A pair $(\omega, A, R, G ; i)$ is a right admissible configuration if $(\omega, A, R, G)$ is a well colored configuration and $i \in A \cup\{\perp\}$, or if $(\omega, A \backslash\{i+1\}, R, G)$ is a well colored configuration and the local configuration between walls $i$ and $i+2$ is $\left.{ }_{i}^{\circ}{ }^{\circ}\right|_{0} ^{\circ} \mid$. Left admissible configurations are defined similarly with $\top$ replaced by $\perp$.

We are now ready to describe a mapping $\psi$, that maps right admissible configurations onto left admissible ones. The image $\left(\omega^{\prime}, A^{\prime}, R^{\prime}, G^{\prime} ; r^{\prime}\right)$ of a right admissible configuration $(\omega, A, R, G ; r)$ by $\psi$ is obtained by applying some local operations near the pointer.

The value $T$ serves as a initialization case: the image of a $\operatorname{pair}(\omega, A, R, G$; $\top)$ by $\psi$ is $(\omega, A, R, G$; $\max (A))$ if $A \neq \emptyset$, and $(\omega, A, R, G ; \perp)$ otherwise. When the pointer $i$ is in $A$, the image $\left(\omega^{\prime}, A^{\prime}, R^{\prime}, G^{\prime} ; i^{\prime}\right)$ of $(\omega, A, R, G ; i)$ depends on the local configuration around $i$, and, if it exits, on the rightmost active wall $m<i$ on the left of $i$ :

A. Cases $: \|_{i \circ}^{\circ}$, or $\bullet_{i}^{\circ} \|^{\circ}$ with $i$ green, or $\|_{0}^{\circ}$ with $i=0:$ (see Figure 5)

Two operations are performed on $\omega$ to produce its image $\omega^{\prime}$. The first one is a left deletion at $i$. For the second let $j_{2}>i$ be the rightmost wall such that there are only black particles in the top row between walls $i+1$ and $j_{2}$. The second operation is a left insertion at $j_{2}$. There are two possibilities:

- The wall $j_{2}$ was not active $\left(j_{2} \notin A\right)$. The wall $j_{2}$ replaces the wall $i$ in the set of active walls: $A^{\prime}=A \cup\left\{j_{2}\right\} \backslash\{i\}$. Moreover, if there is a black particle on its bottom right in $\omega$, the wall $j_{2}$ is colored green: $G^{\prime}=G \cup\left\{j_{2}\right\} \backslash\{i\}$.

- The wall $j_{2}$ was active $\left(j_{2} \in A\right)$. Then $i$ is removed from the set of active walls and the wall $j_{2}$ is colored red: $A^{\prime}=A \backslash\{i\}, R^{\prime}=R \cup\left\{j_{2}\right\}, G^{\prime}=G \backslash\{i\}$.

Other colorings are left unchanged, and in both cases the pointer is set to $m$ if it exists, to $\perp$ otherwise.

B. Cases $\stackrel{\bullet}{?}_{i}^{\circ} \stackrel{\circ}{\bullet}$, or $:_{\circ} \|_{n}$ with $i=n:($ see Figure 6$)$

Two operations are performed on $\omega$ to produce its image $\omega^{\prime}$. The first one is a right deletion at $i$. To describe the second operation, let $j_{1}<i$ be the leftmost wall on the left of $i$ in $\omega$ such that there are only white particles in the top row between walls $j_{1}$ and $i-1$. There are again several possibilities:

- The wall $j_{1}$ was active (so that $m=j_{1}$ ). Then the second operation is a right insertion at wall $m+1$, which becomes active: $A^{\prime}=A \cup\{m+1\} \backslash\{i\}$. (This is the only case in which a wall with local configuration olo can become active. As prescribed in the definition of admissible configuration, the pointer will be set on the left of this abnormal active wall.)

- Otherwise, the second operation is a right insertion at the wall $j_{1}$. The wall $j_{1}$ replaces the wall $i$ in the set of active walls: $A^{\prime}=A \cup\left\{j_{1}\right\} \backslash\{i\}$.

Other colorings are left unchanged, and in all cases the pointer is set to $m$ if it exists and to $\perp$ otherwise.

C. Case $\stackrel{\bullet}{\bullet} \|_{i}^{\circ}$ with $i$ red: (see Figure 7)

Three operations are performed on $\omega$ to produce its image $\omega^{\prime}$. The first one consists in removing the block $: \|_{0}^{\circ}$ around $i$. The second operation is a left insertion at $j_{2}$ as for the cases of type A above, with the same two possibilities for the changes of colors. The third operation is a right insertion at $m+1$ or $j_{2}$ as for the cases of type B above. Again the pointer is set to $m$ if $m$ exists and to $\perp$ otherwise. 

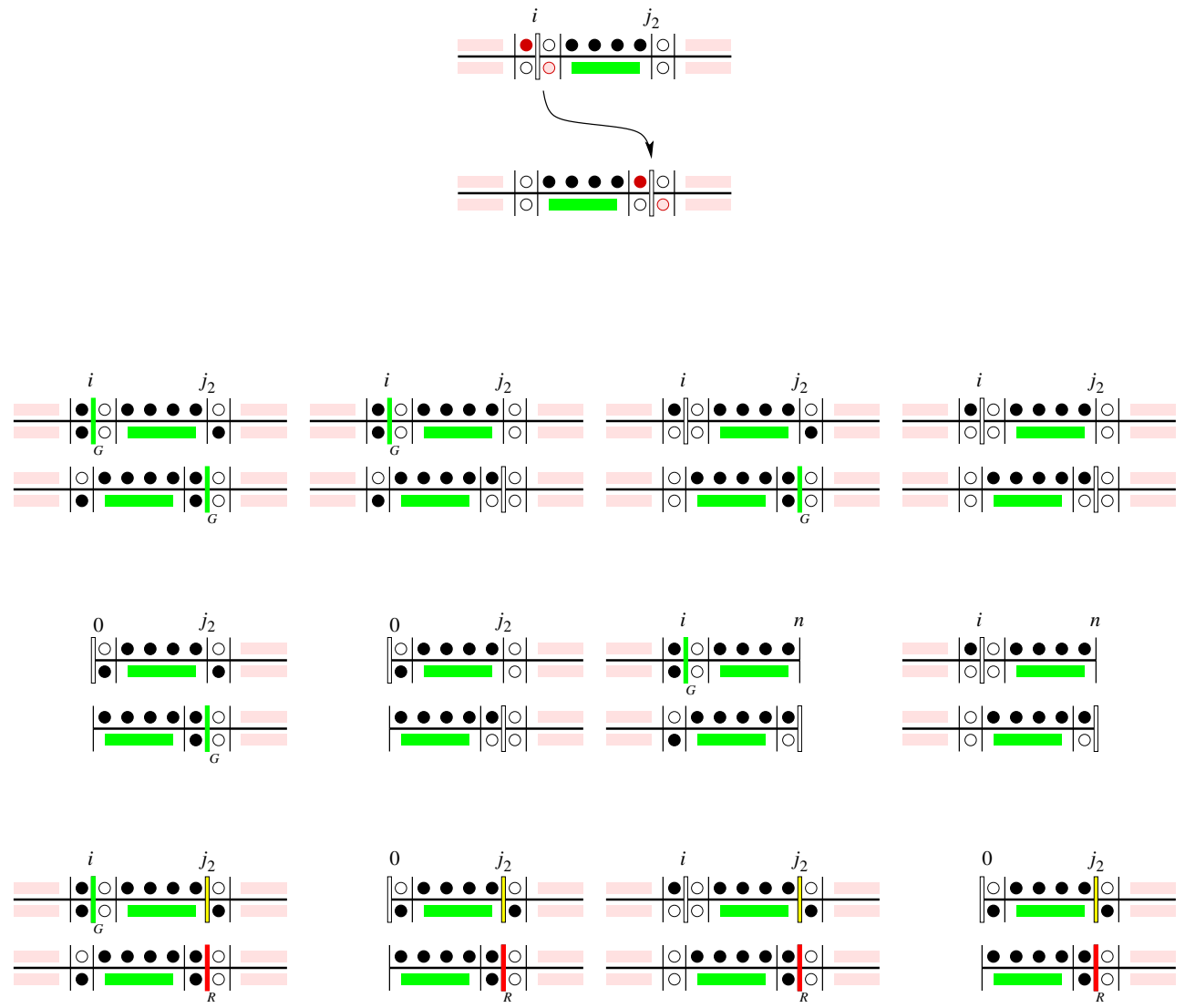

Figure 5. The application $\psi$ in cases of type A. The rule is first presented informally. It is then instantiated for all possible local configurations to allow for easy verification of proofs.
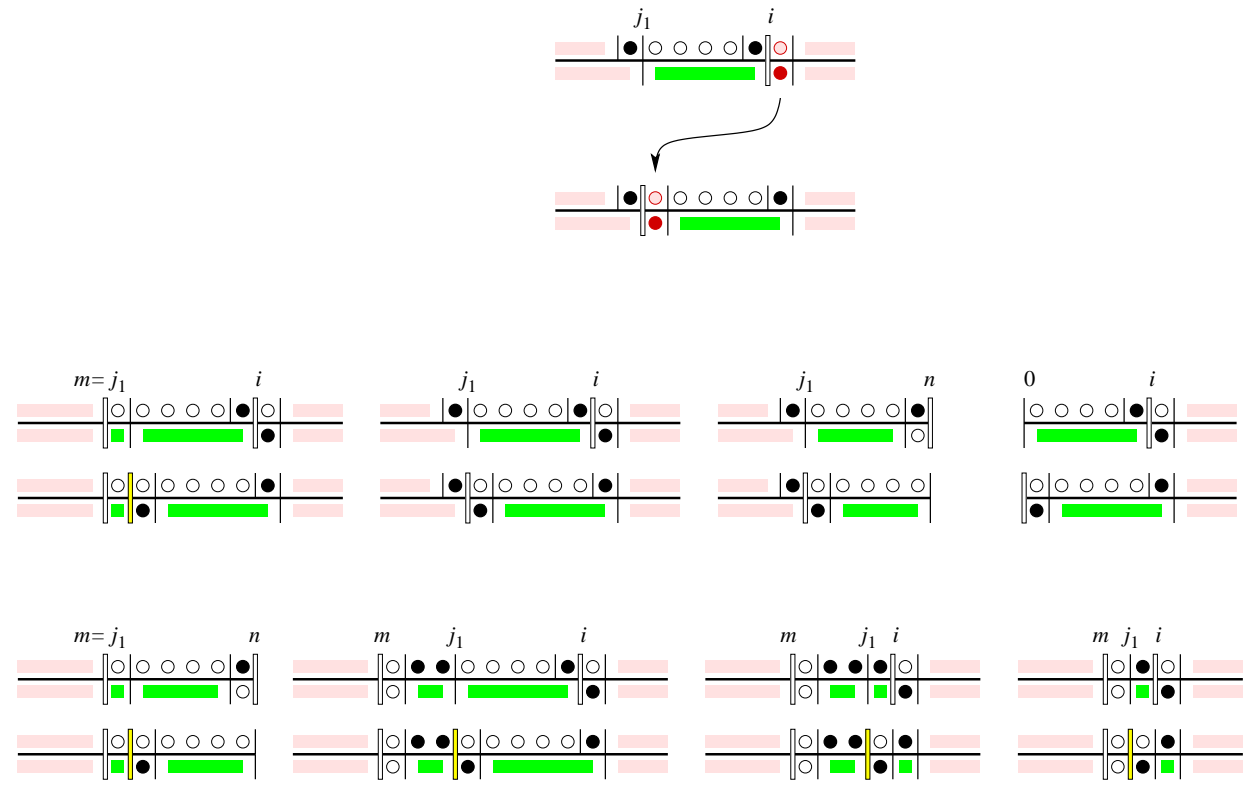

Figure 6 . The application $\psi$ in cases of type B. The rule is first presented informally. It is then instantiated for all possible local configurations to allow for easy verification of proofs. 

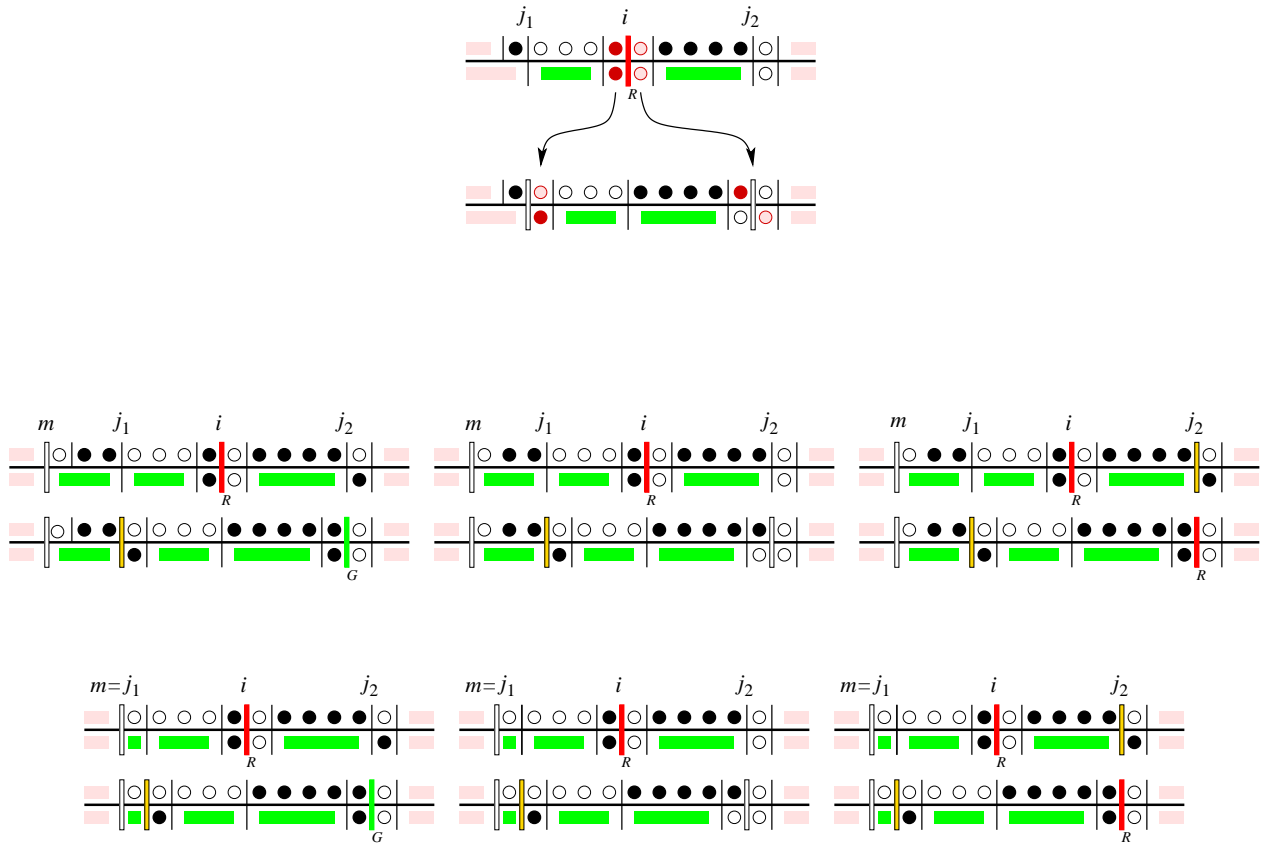

Figure 7 . The application $\psi$ in cases of type C. The rule is first presented informally. It is then instantiated for a selection of possible local configurations. (All configurations can be retrieved by combining type A to the right and type B to the left.)

Lemma 1. The mapping $\psi$ is a bijection from the set of right admissible configurations onto the set of left admissible configurations.

Proof. With the help of Figures 5, 7 and 6, one can easily check the following properties, which together prove the lemma.

First the image of a right admissible configuration by $\psi$ is a left admissible configuration. The fact that the image configuration $\omega^{\prime}$ satisfies the positivity condition follows immediately from the fact that the local operations of Section 3.1 preserve this condition. It should be observed also that if there is an abnormal active wall on the right of the pointer in $\omega$ (i.e. an active wall with local configuration o/o in the top row), then the application of $\psi$ brings a black particle to its left, hence turning it back into a normal active wall.

Second a case analysis allows to check that any left admissible configuration has a preimage by $\psi$.

3.3. The bijection $\bar{T}$. Observe that the mapping $\psi$ always moves the pointer to the left. The mapping $\bar{T}$ is defined by iterating $\psi$ so that the pointer goes from $\top$ to $\perp$.

- Let $\ell:=0$ and $\left(\omega_{0}, A_{0}, R_{0}, G_{0}\right)=(\omega, A, R, G)$, and set the initial pointer to the right of the configuration: $i_{0}=\top$.

- Repeat

- let $\left(\omega_{\ell+1}, A_{\ell+1}, R_{\ell+1}, G_{\ell+1} ; i_{\ell+1}\right)=\psi\left(\omega_{\ell}, A_{\ell}, R_{\ell}, G_{\ell} ; i_{\ell}\right)$ and $\ell:=\ell+1$.

until $i_{\ell}$ reaches the value $\perp$.

- The image $\bar{T}(\omega, A, R, G)$ is $\left(\omega_{\ell}, A_{\ell}, R_{\ell}, G_{\ell}\right)$.

In view of the properties of the application $\psi$, the following is immediate.

Proposition 2. The mapping $\bar{T}$ is a bijection from the set of well colored configurations onto itself. Moreover if $\left(\omega^{\prime}, A^{\prime}, R^{\prime}, G^{\prime}\right)=\bar{T}(\omega, A, R, G)$, then $\omega^{\prime}=\vartheta(\omega, A)$.

Although it is more convenient to describe $\bar{T}$ in a sequential way, as we did in terms of $\psi$, it is worth observing again that $\bar{T}$ essentially acts in parallel on all walls, with one pair of particles leaving or arriving at every non-red active wall, and two pairs of particles leaving or arriving at every red active wall. 
Another remarkable feature of the bijection $\bar{T}$ is that it can be interpreted as moving black particles to the right in the top row and in the opposite direction in the bottom row. Indeed, as can be checked on Figure 5, 6 , and 7 , each time a green region is moved to the right, a black particle is put on its left, so that the global move can be reinterpreted as the displacement of some black particles to the left. The reader is referred to [3] where a similar interpretation in terms of circulating particles is developed for the sequential case.

3.4. The bijection and the parameter. In order to conclude the proof of Proposition 1, it remains to check that for any well colored configuration $\left(\omega, A, A^{\prime}, A^{\prime \prime}\right)$ we have

$$
q\left(\bar{T}\left(\omega, A, A^{\prime}, A^{\prime \prime}\right)\right)=q\left(\omega, A, A^{\prime}, A^{\prime \prime}\right) .
$$

In order to do so, it is sufficient to prove that the parameter $q$ is left unchanged by the application $\psi$. This can be easily be checked on Figures 5, 6 and 7, since all possibilities have been explicitly listed. (The only difficulty is not to forget to count the contribution of all walls of $A$, even the one that does not belong to $M(\omega)$ when it exists.)

\section{Conclusion}

The interest in the parallel TASEP originates in the Nagel-Schreckenberg automaton, which is a landmark of highway traffic flow modeling.

The Nagel-Schreckenberg automaton. A configuration of this Markov chain consists of a row of $n$ cells and $n+1$ walls containing some cars. Each cell can be occupied by a car and cars are numbered from left to right. The $j$ th car is characterized at time $t$ by its position $x_{j}$ and its velocity $v_{j}(t) \in\left\{0,1, \ldots, v_{\max }\right\}$, where $v_{\max }$ is the maximal velocity chosen for the system. Moreover, let us denote by $d_{j}(t)$ the distance, i.e. the number of cells between the $j$ th car and the $(j+1)$ th car.

- At time $t=0$, the system is in a configuration $N S(0)$ (possibly chosen at random).

- From time $t$ to $t+1$, the system evolves from the configuration $N S(t)$ to the configuration $N S(t+1)$ by applying the following successive transformations to all cars in parallel (see Figure 8):

A Acceleration. If the $j$ th car is not at the maximal velocity then its velocity increases by one, i.e. $v_{j}^{\prime}(t)=\min \left(v_{j}(t)+1, v_{\max }\right)$.

D Safety deceleration. If the distance $d_{j}(t)$ to the next car is less than its velocity $v_{j}^{\prime}(t)$ then the latter decreases to $d_{j}(t)$, i.e. $v_{j}^{\prime \prime}(t)=\min \left(d_{j}(t), v_{j}^{\prime}(t)\right)$.

$\mathbf{R}$ Random deceleration. The $j$ th car can decelerate by one with probability $q$ if $v_{j}^{\prime \prime}(t)$ is not zero:

$$
v_{j}(t+1)= \begin{cases}\max \left(v_{j}^{\prime \prime}(t)-1,0\right) & \text { with probability } q \\ v_{j}^{\prime \prime}(t) & \text { otherwise. }\end{cases}
$$

M Movement. The $j$ th car moves $v_{j}(t+1)$ cells to the right, i.e. $x_{j}(t+1)=x_{j}(t)+v_{j}(t+1)$. The resulting $\left(x_{j}(t+1), v_{j}(t+1)\right)_{j}$ define the new configuration $N S(t+1)$, and in particular the new distances $d_{j}(t+1)$.

Although the Nagel-Schreckenberg automaton is a Markov chain with very simple rules, it appears to be difficult to study from a mathematical point of view. In particular, its stationary distribution is only known for the particular case $v_{\max }=1$ : indeed if $v_{\max }=1$ then the Nagel-Schreckenberg automaton corresponds to the parallel TASEP, where cars are black particles, and where $q=1-p$. Indeed, when $v_{\max }=1$, the possible velocities for each car are 0 or 1 . Therefore, after step $\mathbf{A}$ all the velocities are equal to 1 . At step $\mathbf{D}$ only cars with $d_{j}=0$ (i.e. immediately followed by another car) decrease their velocity to 0 . At step $\mathbf{R}$ a car $j$ such that $v_{j}=1$ keeps its non zero velocity with probability equal to $1-q=p$. At step $\mathbf{M}$ only cars with $d_{j} \neq 0$ and $v_{j}=1$ move of one cell to the right. This is equivalent to say that from time $t$ to time $t+1$ cars that have a free cell on their right can move in there with probability $p$. This corresponds exactly to the parallel TASEP.

A challenging open problem is of course to compute the stationary distribution of the Nagel-Schreckenberg automaton for larger velocities. 


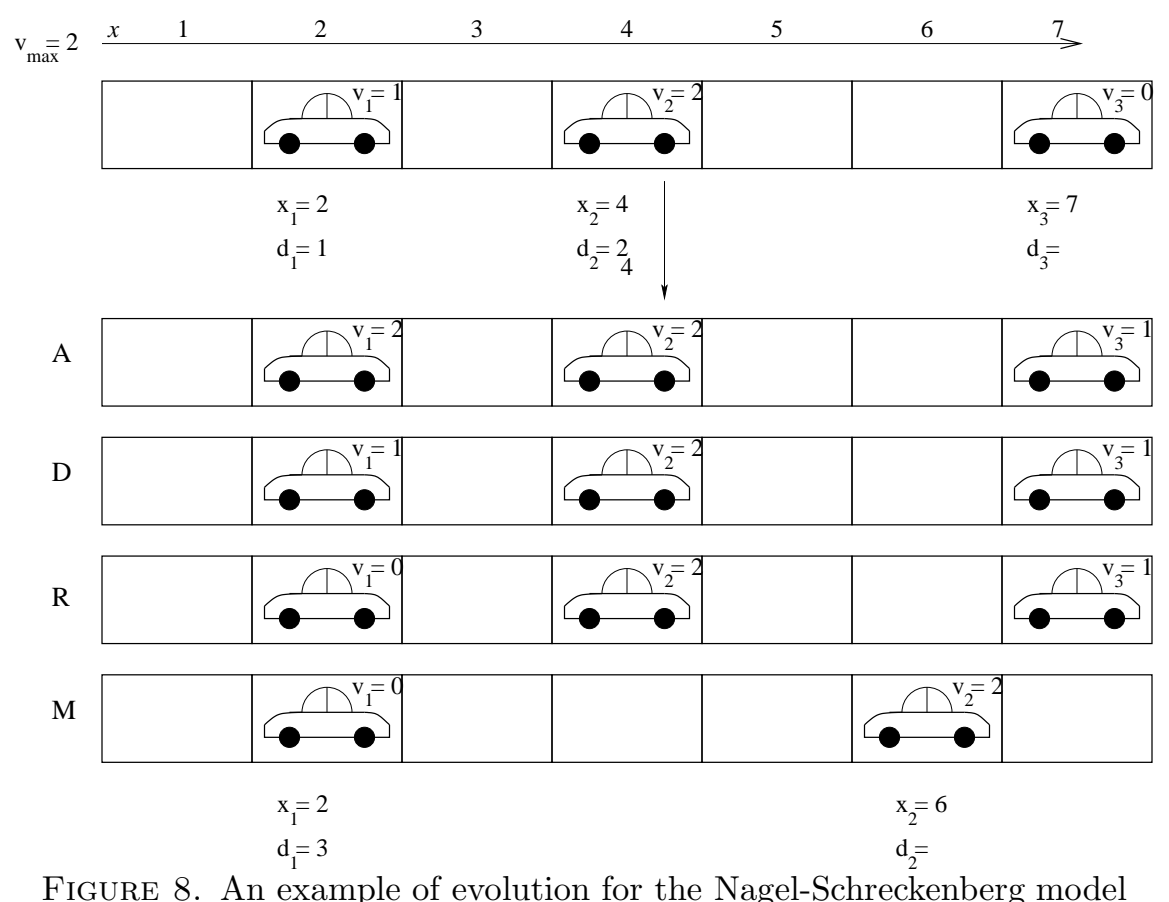

\section{REFERENCES}

[1] B. Derrida, E. Domany, and D. Mukamel. An exact solution of a one dimensional asymmetric exclusion model with open boundaries. J. Stat. Phys., 69:667-687, 1992.

[2] B. Derrida, M.R. Evans, V. Hakim, and V. Pasquier. Exact solution of a one-dimensional asymmetric exclusion model using a matrix formulation. J. Phys. A: Math., 26:1493-, 1993.

[3] E. Duchi and G. Schaeffer. A combinatorial approach to jumping particles. To appear in Journal of Combinatorial Theory, series A, 2004.

[4] M. R. Evans, N. Rajewsky, and E. R. Speer. Exact solution of a cellular automaton for traffic. Journal of Statistical Physics, 95(1-2):45-96, 1999.

[5] O. Häggström. Finite Markov Chains and Algorithmic Applications. Cambridge University Press, 2002.

[6] A. Schadschneider. Traffic flow: A statistical physics point of view. Physica A, 313:153, 2002.

Enrica Duchi, LiAfA, Paris 7, France

E-mail address: mailto:Enrica.Duchi@liafa.jussieu.fr

Gilles Schaeffer, LiX, CNRS, France

E-mail address: mailto:Gilles.Schaeffer@lix.polytechnique.fr 